Abstract P216 Table 1 Eligibility rates and reasons for exclusion identified in two COPD inpatient studies

\begin{tabular}{|c|c|c|}
\hline & Frequency $1^{\circ}$ & \\
\hline & $\begin{array}{l}\text { Clinical trial } \\
\left(\mathrm{n}=71^{*}\right)\end{array}$ & $\begin{array}{l}\text { Observational } \\
\text { study ( } \mathrm{n}=172 \text { ) }\end{array}$ \\
\hline Eligible for inclusion & $8(11)$ & $48(29)$ \\
\hline Reasons for exclusion & & \\
\hline Cardiovascular & $8(11)$ & $13(8)$ \\
\hline Respiratory & $8(11)$ & $18(10)$ \\
\hline Neurological (incl. dementia, delirium) & $7(10)$ & $28(16)$ \\
\hline Renal & $8(11)$ & $0(0)$ \\
\hline Gastrointestinal and nutrition & $6(8)$ & $0(0)$ \\
\hline Endocrine and metabolic (incl. diabetes) & $10(14)$ & NC \\
\hline Malignancy & NC & $23(13)$ \\
\hline Frailty & NC & $19(11)$ \\
\hline Drug / alcohol misuse & $1(1)$ & $13(8)$ \\
\hline Language / literacy & NC & $10(6)$ \\
\hline
\end{tabular}

${ }^{*}$ Some patients had more than one reason for exclusion.

$\mathrm{NC}$, not collected

Conclusion Patients hospitalised for COPD exacerbations are heterogeneous and have significant and diverse co-morbidities which may limit their eligibility for research studies. In view of this, broad entry criteria are necessary to ensure that studies in this population are feasible. With careful design, such studies will be pivotal in driving improved treatment and outcomes among these patients who, despite their poor prognosis, presently receive disproportionately little research attention.

\section{P217 FACTORS AFFECTING PATIENT SATISFACTION IN A COPD RESEARCH COHORT}

doi:10.1136/thoraxjnl-2011-201054c.217

B S Kowlessar, A R C Patel, G C Donaldson, A J Mackay, J R Hurst, J A Wedzicha. Academic Unit of Respiratory Medicine, UCL Medical School, London, UK

Background Measures of patient satisfaction have become increasingly important in modern healthcare and clinical research. We aimed to assess and quantify factors relating to patient satisfaction in the London COPD cohort.

Methods Questionnaires were posted to patients in the London COPD cohort (anonymised to clinic staff) including categorical items (Likert scale or Yes/No) and freetext boxes. Data were analysed from the last stable state visit before October 2010 including demographics, spirometry, MRC dyspnoea, and St George's Respiratory Questionnaire (SGRQ) scores. Data were analysed using Spearman's rank correlation, Mann-Whitney $U$ and $\chi^{2}$ tests.

Results 130 respondents (response rate $68 \%$ ) had a mean $( \pm \mathrm{SD})$ age of $73.4( \pm 8.8)$ years and mean $\mathrm{FEV}_{1} 50.6 \%$ ( $\pm 19.3 \%$ ) predicted. $55 \%$ were male, $26 \%$ were current smokers with median (IOR) pack year history of 47 (26-73). There were no significant differences between respondents and non-respondents. $96 \%$ of respondents reported that they were very satisfied $(74 \%)$ or satisfied $(22 \%)$ with the COPD research (Abstract P217 figure 1). Satisfaction correlated with providing prompt and appropriate treatment at exacerbation onset $(\mathrm{r}=0.419, \mathrm{p}<0.001)$ and during routine visits $(\mathrm{r}=0.577, \mathrm{p}<0.001)$. Satisfaction also correlated with several staff-related factors: courteousness $(r=0.545, p<0.001)$, being easy to contact $(r=0.498$, $\mathrm{p}<0.001)$, providing advice on symptom diaries $(\mathrm{r}=0.553, \mathrm{p}<0.001)$, explaining exacerbation recognition $(\mathrm{r}=0.507, \mathrm{p}<0.001)$, explaining research investigations $(r=0.622, p<0.001)$, feeding back individual results $(r=0.476, p<0.001)$, and overall research findings $(r=0.409$, $\mathrm{p}<0.001)$. It also correlated with patients feeling more confident managing their COPD and exacerbations $(r=0.465, p<0.001)$. Patients who were in the cohort for longer were more likely to be more satisfied $(\mathrm{r}=0.289, \mathrm{p}=0.001)$. There was no correlation between satisfaction and age, gender, spirometry, smoking, BMI, SGRO or MRC dyspnoea scores. 94\% reported less anxiety knowing they could call the doctors at any time, 77\% felt they attended A\&E less since joining the cohort, $80 \%$ were less likely to visit their GP at exacerbation.

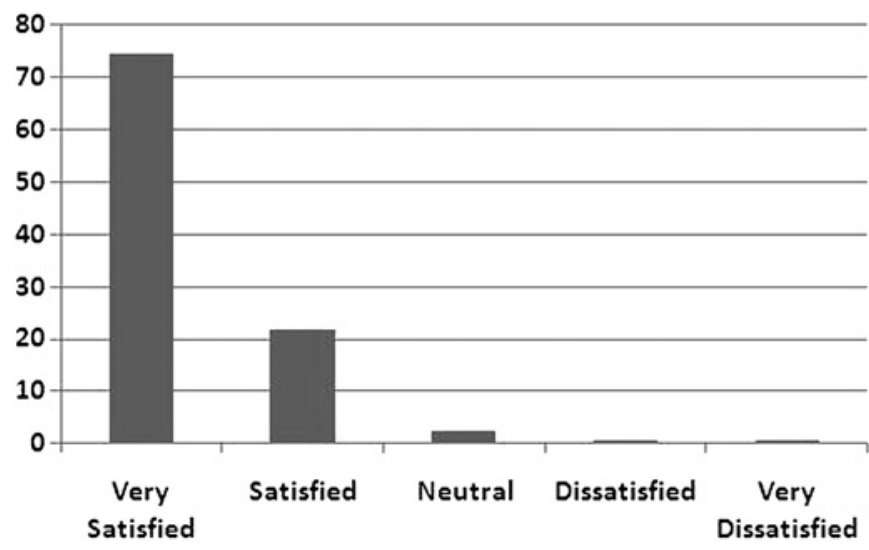

Abstract P217 Figure 1 Percentage of respondents satisfied with research in the London COPD Cohort.

Conclusions Patient satisfaction is very high in the London COPD cohort and was associated with positive staff interactions, prompt exacerbation treatment and detailed explanations. Involvement in the cohort also led most patients to feel less anxious, reportedly attending A\&E and GP services less frequently.

\section{P218 RECORDING OF COPD MORTALITY MUST IMPROVE IF IT IS TO BE A ROBUST OUTCOME MEASURE}

doi:10.1136/thoraxjnl-2011-201054c.218

S Ansari, M Ali, D J Powrie, K G Lingam, A G Davison. Southend University Hospital, Southend on Sea, UK

Statistics for chronic obstructive pulmonary disease (COPD) are dependant on local data collection and have an impact on understanding the morbidity and mortality. This can steer the resources and ought to be robust. Inaccuracies in coding can affect the hospital standardised mortality ratio. The Coalition government's "Outline Strategy for COPD and Asthma in England" published in 2011, emphasises the focus on outcomes. We examined in-patient mortality of COPD at Southend University Hospital to confirm the accuracy of the data. The coding department provided a list of patients coded with COPD who died in the hospital during the study period of 1 year (1 April 2009 and 31 March 2010). All death certificates for the same period were reviewed and those with cause of death recorded as COPD identified. The two lists were compared. Five consultant respiratory physicians reviewed the notes, independently recorded the cause of death and compared to death certificates. According to death certificates 77 patients died of COPD, whereas a total of 55 COPD related deaths were identified by the coding department. The later was compared with actual death certificates for corroboration: COPD as a disease directly leading to death (Ia) was recorded in 21 , as a disease leading to $1 \mathrm{a}$ (Ib) in 11, as a disease leading to $1 \mathrm{~b}$ (Ic) in none and as another significant condition contributing to death (II) in 8. Two were referred to the coroner, no data available for two and no COPD was recorded in the remaining 11 . Of the available 41 notes (from the coding department's list) reviewed by respiratory consultants, COPD as a cause of death in their view was Ia in 13 , Ib in 5 , Ic in none and II in 10 cases and it was not the cause in the rest. Mortality data from coding, death certification and opinion of 